\title{
Physiopathologie de la sclérodermie systémique
}

\section{Yannick Allanore}

> La sclérodermie systémique (SSc) est une maladie orpheline atteignant le tissu conjonctif. L'origine de la maladie n'est pas connue mais elle implique probablement des facteurs environnementaux sur un fond génétique de susceptibilité. La physiopathologie est dominée par des lésions microvasculaires précoces responsables de lésions endothéliales avec la production de nombreux médiateurs favorisant une réponse inflammatoire et un remodelage vasculaire. Beaucoup d'arguments mettent en avant des phénomènes d'auto-immunité pour entretenir et perpétuer le processus pathologique. La cascade aboutit à des lésions de fibrose définies par l'accumulation de substances de la matrice extracellulaire en raison de perturbations de l'équilibre synthèse/dégradation de nombreux composants, une activation et une différenciation de cellules mésenchymateuses liées à des facteurs autocrines et paracrines. <

La sclérodermie systémique (SSc, systemic sclerosis) fait partie des maladies fibrosantes chroniques dont l'origine n'est pas connue. Elle présente plusieurs originalités et spécificités dont une microangiopathie qui a une expression clinique particulière et une importante composante auto-immune. Elle est unique par son atteinte dermatologique qui lui a donné son nom skleros (dur) et derma (derme). Celle-ci constitue une composante clinique fondamentale, souvent prédominante, mais qui peut être régressive. En effet, après une phase de progression, on peut observer, chez certains malades, une désinfiltration cutanée spontanée. Classiquement, la fibrose est considérée comme irréversible. Le modèle de la SSc est ainsi très intéressant pour explorer les processus régulateurs qui contribuent à la formation et à la résorption d'une fibrose.

Une avancée majeure des dernières années réside dans la validation de nouveaux critères de classification des différentes formes de sclérodermie systémique (Tableaux / et II) [1]. Alors que les anciens critères

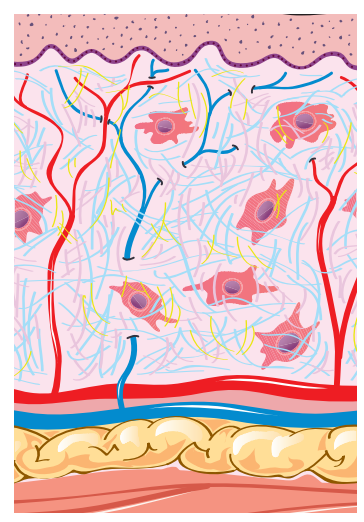

Inserm U1016, institut Cochin, rhumatologie $A$, université Paris Descartes, hôpital Cochin, 27, rue du Faubourg-SaintJacques, 75014 Paris, France. yannick.allanore@inserm.fr

identifiaient plutôt des formes avancées de la maladie, il s'agit désormais d'identifier les malades à un stade où les lésions sont encore précoces et majoritairement réversibles. Cette démarche de reclassification, qui ouvre la voie à des traitements précoces, a notamment été appliquée à la polyarthrite rhumatoïde et a permis d'immenses progrès dans la prise en charge de la maladie. Elle a conduit à créer le concept de fenêtre d'opportunité thérapeutique. Les nouveaux critères de la SSc permettent de porter le diagnostic chez un malade présentant un syndrome de Raynaud ${ }^{1}$, des auto-anticorps spécifiques, des anomalies à la capillaroscopie périunguéale $e^{2}$ et une atteinte dermatologique (œdémateuse, fibreuse ou vasculaire) même minime (Figure 1). La SSc est associée à une surmortalité significative [2]. Des progrès sont donc nécessaires, et la constitution d'un groupe de recherche européen, dont un des outils est une base de données qui comporte plus de 12000 cas référencés, est un réel espoir pour mieux comprendre la maladie et développer des approches thérapeutiques innovantes [3]. La SSc est un modèle de maladie multifactorielle qui implique des facteurs environnementaux et un fond génétique de susceptibilité. La séquence pathogénique associe des désordres vasculaires (microangiopathie), inflammatoires et immunologiques (auto-immunité) et des signaux profibrosants (Figure 2). De nombreux médiateurs ayant une action paracrine ou autocrine interviennent, en particulier le TGF $\beta$ (transforming growth factor $\beta$ ) qui a un rôle de premier plan. Toutefois, les interactions entre les différents acteurs cellulaires et moléculaires sont encore mal élucidées mais elles pourraient jouer un rôle clé dans l'extrême hétérogénéité qui caractérise cette maladie.

${ }^{1}$ Le syndrome de Raynaud est un trouble de la circulation sanguine se manifestant par un spasme artériolaire, conduisant à des phases successives blanches, violacées puis rouges avec engourdissement ou des douleurs, affectant les extrémités comme les doigts, les orteils, le nez, ou les oreilles.

${ }^{2}$ La capillaroscopie périunguéale est une méthode d'exploration non invasive des capillaires du derme superficiel, effectuée par microscopie optique de faible grandissement. 


\section{Critère majeur}

Sclérose cutanée s'étendant au-dessus des articulations métacarpophalangiennes ou métatarso-phalangiennes (touchant les avant-bras, les bras, les jambes, les cuisses, les doigts étant quant à eux pratiquement toujours concernés), et touchant aussi la face, le cou, le tronc.

\section{Critères mineurs}

- Sclérodactylie (épaississement cutané limité aux doigts et aux orteils),

- Cicatrice digitale ou perte de substance de la pulpe ou de l'extrémité du doigt,

- Fibrose pulmonaire des bases.

1 critère majeur ou au moins 2 critères mineurs sont nécessaires pour affirmer le diagnostic

Tableau I. Critères diagnostiques de la sclérodermie systémique définis en 1980, selon la société américaine de rhumatologie.

\section{Atteinte vasculaire et microangiopathie}

L'atteinte du réseau microvasculaire et l'activation des cellules endothéliales semblent être parmi les événements les plus précoces de la maladie [4]. L'activation des cellules endothéliales conduit à l'expression de molécules d'adhésion (dont ICAM [intercellular adhesion molecule], VCAM [vascular cell adhesion molecule], des sélectines) qui favorisent le recrutement des cellules inflammatoires [5]. La synthèse de modulateurs du tonus vasculaire est également perturbée avec, notamment, un excès de synthèse d'endothéline et une moindre production de monoxyde d'azote et de prostacyclines, ce qui perturbe les fonctions de contrôle du tonus et de la perméabilité vasculaires, et l'action antithrombotique physiologique de l'endothélium. Les cellules endothéliales produisent, de plus, des molécules profibrosantes dont l'endothéline et le CTGF (connective tissue growth factor également appelé (CN2), qui stimulent la prolifération des cellules musculaires lisses et la synthèse de composants de la matrice extracellulaire (MEC) [6]. Les cellules inflammatoires qui sont recrutées au niveau des sites agressés, semblent par ailleurs être une source importante de cytokines pro-inflammatoires (IL[interleukine]-l et IL-6) et de molécules profibrosantes (TGF $\beta$, PDGF [platelet-derived growth factor]) (Figure 2). L'évolution des lésions conduit à une raréfaction capillaire, à un épaississement de la paroi des vaisseaux en raison d'une prolifération intimale ${ }^{3}$ et des cellules musculaires

${ }^{3}$ L'intima est la plus interne des trois couches formant la tunique des artères et des veines.

\begin{tabular}{|c|c|c|}
\hline Items & Sous-item(s) & Poids/score \\
\hline $\begin{array}{l}\text { Épaississement cutané des doigts } \\
\text { des deux mains s'étendant jusqu'aux } \\
\text { articulations métacarpophalangiennes } \\
\text { (critère suffisant) }\end{array}$ & & 9 \\
\hline $\begin{array}{l}\text { Épaississement cutané des doigts } \\
\text { (ne compter que le score le plus élevé) }\end{array}$ & $\begin{array}{l}\text { Doigts boudinés } \\
\text { Sclérodactylie (en distalité des } 4 \text { articulations métacarpophalangiennes, } \\
\text { mais proximale jusqu'aux articulations interphalangiennes) }\end{array}$ & $\begin{array}{l}2 \\
4\end{array}$ \\
\hline $\begin{array}{l}\text { Lésions pulpaires } \\
\text { (ne compter que le score le plus élevé) }\end{array}$ & $\begin{array}{l}\text { Ulcères pulpaires } \\
\text { Cicatrices pulpaires déprimées }\end{array}$ & $\begin{array}{l}2 \\
3\end{array}$ \\
\hline Télangiectasies & - & 2 \\
\hline Anomalies capillaroscopiques & - & 2 \\
\hline $\begin{array}{l}\text { Hypertension artérielle pulmonaire } \\
\text { et/ou atteinte pulmonaire interstitielle } \\
\text { (score maximum 2) }\end{array}$ & $\begin{array}{l}\text { Hypertension artérielle pulmonaire } \\
\text { Atteinte pulmonaire interstitielle }\end{array}$ & $\begin{array}{l}2 \\
2\end{array}$ \\
\hline Phénomène de Raynaud & - & 3 \\
\hline $\begin{array}{l}\text { Autoanticorps associés à la SSc } \\
(\text { score maximum }=3 \text { ) }\end{array}$ & $\begin{array}{l}\text { Anti-centromères } \\
\text { Anti-topo-isomérase I } \\
\text { Anti-ARN polymérase III }\end{array}$ & $\begin{array}{l}3 \\
3 \\
3\end{array}$ \\
\hline
\end{tabular}

Tableau II. Critères de classification ACR/EULAR (American college of rheumatology et European ligue against rheumatism) définis en 2013 pour la sclérodermie systémique. Télangiectasies : dilatation des petits vaisseaux cutanés; anomalies capillaroscopiques : la capillaroscopie montre une image anormale (méga-capillaires, hémorragies ou plages désertes). Un score de 9 ou plus est nécessaire à la classification d’un malade. 


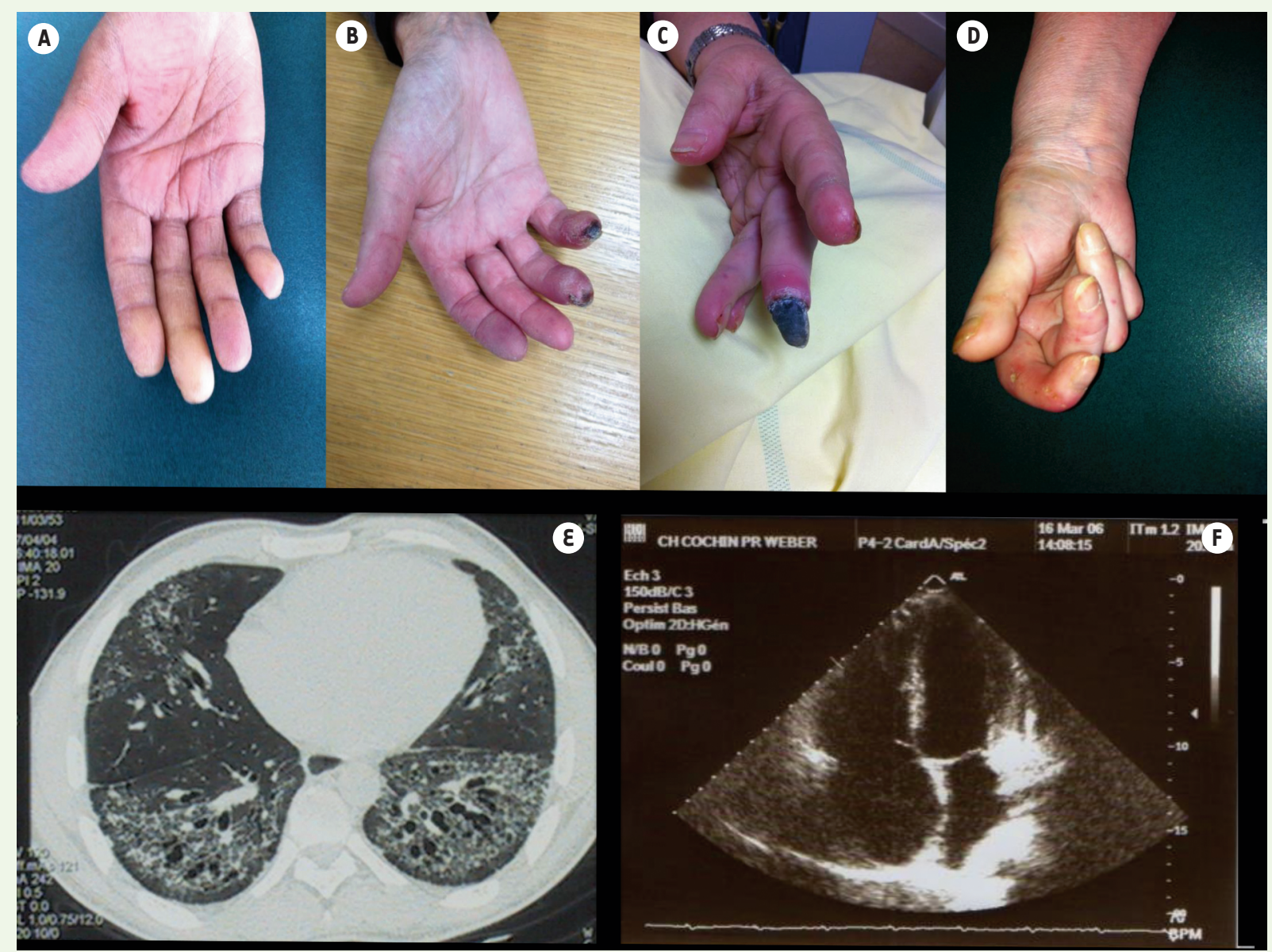

Figure 1. Caractéristiques cliniques. Syndrome de Raynaud avec spasme des doigts et phase ischémique notamment du majeur (A), puis aggravation des troubles vasculaires avec nécrose digitale $(B)$ et gangrène $(C)$. Lésions déformantes sévères intégrant une sclérose cutanée majeure et des déformations articulaires ( $D$ ) avec aspect évocateur de rétraction digitale. Lésions d'organe internes avec fibrose pulmonaire $(\varepsilon)$ et dilatation des cavités cardiaques droites dans le cadre d'une hypertension pulmonaire (F).

lisses, et finalement à l'oblitération des petits vaisseaux qui est responsable d'une hypoxie et d'un stress oxydant.

L'hypertension artérielle pulmonaire (HTAP) est l'une des conséquences les plus sévères de cette vasculopathie, le remodelage des petits vaisseaux pulmonaires conduisant à une augmentation des résistances pulmonaires puis à une insuffisance cardiaque [7]. La réduction du facteur transcriptionnel GATA-6 (GATA-binding factor 6), qui précède les occlusions vasculaires, témoignerait d'une phase précoce d'activation des cellules endothéliales et/ou de leur dysfonctionnement. Ainsi, des souris dont le gène Gata-6 a été invalidé sélectivement dans leurs cellules endothéliales, développent spontanément des signes d'HTAP [8].

Un autre acteur important est le VEGF (vascular endothelial growth factor), dont la synthèse est stimulée par la perméabilité endothéliale accrue et les phénomènes d'hypoxie. Son action qui pourrait représenter une tentative de réparation des dégâts vasculaires, est cependant insuffisante et/ou inefficace ; ceci peut être illustré par l'augmentation des concentrations sériques de VEGF corrélée à la présence de plages avasculaires (visualisées en capillaroscopie périunguéale). Le VEGF pourrait également représenter un lien moléculaire entre l'atteinte vasculaire et la fibrose. En effet, les signes caractéristiques de I'HTAP de la SSc ont pu être reproduits dans un modèle murin (les souris $T \beta R I I \Delta k$-fib exprimant un récepteur de type 2 au TGF $\beta$ non fonctionnel sous le contrôle d'un promoteur spécifique des fibroblastes) après inhibition du VEGF, soulignant les liens entre les voies du TGF $\beta$ et celles du VEGF [9]. II semble également exister dans la SSc une altération des mécanismes compensatoires de l'atteinte vasculaire que sont à la fois l'angiogenèse (croissance compensatrice de nouveaux vaisseaux à partir des vaisseaux résiduels) et la vasculogenèse (formation de nouveaux vaisseaux de novo). L'hypothèse de l'implication du VEGF dans cette perturbation de l'angiogenèse, dans le contexte de fibrose, est renforcée par la 


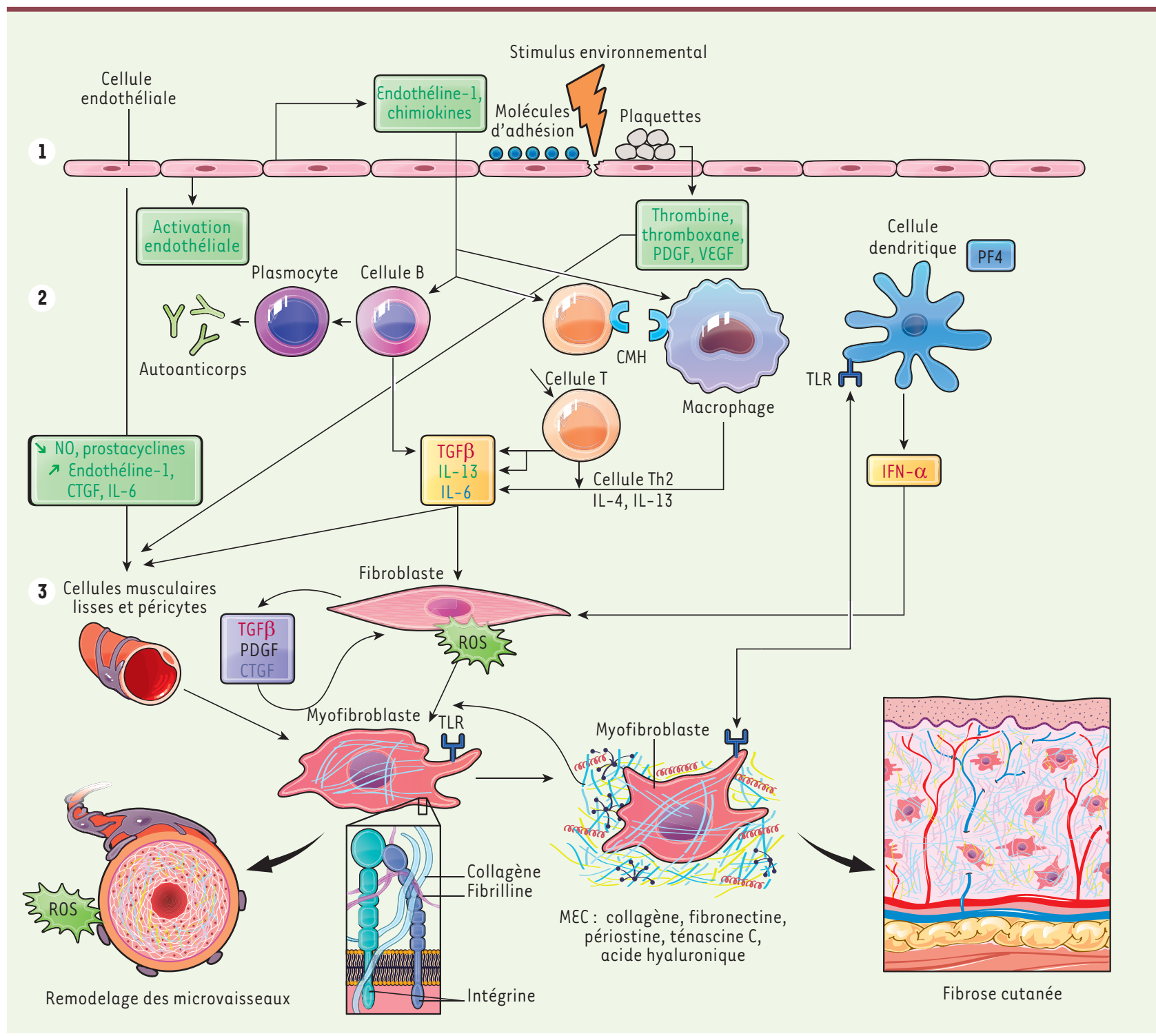

Figure 2. Synthèse physiopathologique. L'activation fibroblastique qui conduit à la fibrose caractéristique de la SSc résulte de lésions microvasculaires avec agression endothéliale accompagnée d'une réaction inflammatoire et immunitaire dérégulée. Étape 1. Activation endothéliale, perméabilité, expression de molécules d'adhésion et dépôt de plaquettes conduisent à la synthèse de vasomodulateurs, de cytokines, de facteurs de croissance et de chimiokines. Étape 2. Différentes cellules inflammatoires et immunitaires sont recrutées et activées. Elles produisent de l'interféron de type 1, des cytokines Th2, de l'interleukine 6, des facteurs de croissance et des autoanticorps. Étape 3. Les fibroblastes sont activés par ces stimulus, produisent de la matrice de façon dérégulée, se différencient en myofibroblastes qui entretiennent le processus avec une matrice désorganisée, des troubles métaboliques en réponse au stress mécanique et à l'hypoxie, une production d'espèces réactives de l'oxygène et de facteurs de croissance conduisant au remodelage vasculaire et à la fibrose tissulaire. PDGF : platelet-derived growth factor ; VEGF : vascular endothelial growth factor; CMH : complexe majeur d'histocompatibilité ; CTGF : connective tissue growth factor ; TGF $\beta$ : transforming growth factor-beta ; TLR : toll-like receptor ; MEC : matrice extracellulaire ; PF4 : platelet factor 4 ; IFN- $\alpha$ : interféron alpha ; ROS : espèces réactives de l'oxygène ; IL : interleukine; NO : monoxyde d'azote.

mise en évidence, dans des souris transgéniques $V \varepsilon G F^{+/-}$, d'un plus grand nombre de microvaisseaux, comparées à des animaux $V \varepsilon G F^{+/+}$, qui expriment VEGF et développent spontanément une fibrose [10]. L'expression d'isoformes antiangiogéniques du VEGF et la perte d'expression du facteur cellulaire endothélial EGFL7 (epidermal growth factor like domain 7) ${ }^{4}$ dans les cellules endothéliales et leurs progéniteurs, ont été suggérées. In vivo, il a ${ }^{4}$ La protéine EGF7 est sécrétée par les cellules endothéliales et est associée à la
matrice extracellulaire. 
également été proposé que la capacité de néovascularisation des progéniteurs endothéliaux issus de patients atteints de SSc soit diminuée, participant ainsi au déficit de la vasculogenèse compensatoire [11].

\section{Inflammation et réponse immune}

Des perturbations de l'immunité innée et acquise jouent un rôle clé dans la séquence pathogénique de la SSc. Des « signatures » inflammatoires sont trouvées dans les sites lésionnels, dont la peau et le poumon, et des perturbations du nombre et des fonctions des cellules immunitaires ont été mises en évidence. Ainsi, chez les patients, une signature « interféron de type I » est bien caractérisée dans le sang circulant mais également les tissus lésés. Parallèlement, des autoanticorps sont également détectés chez la majorité des malades atteints de SSc. De même, des variants dans les gènes codant différents acteurs de la réponse immune, ont été identifiés.

\section{Réponse cellulaire}

Les monocytes/macrophages circulants et tissulaires, les cellules dendritiques plasmacytoïdes et les cellules stromales expriment une signature interféron de type 1 qui reflète une activation impliquant les récepteurs de l'immunité innée, les TLR (Toll-like receptors) [12]. Cette activation semble avoir pour origine des ligands endogènes, dont des acides nucléiques, et également des molécules associées aux signaux de danger, les DAMP (damage-associated molecular patterns), comme des composants de la matrice extracellulaire (MEC) libérés en cas de lésions tissulaires. Parmi les TLR, le TLR-4, initialement identifié comme récepteur du lipopolysaccharide bactérien (LPS), reconnaît des molécules constitutives de la MEC comme l'acide hyaluronique (HA), des fragments de fibronectine ou l'héparane sulfate. L'implication du TLR-4 a pu être montrée dans un modèle animal de souris invalidées pour le gène $t$ lr4, chez lesquelles l'exposition à la bléomycine induisait une moindre fibrose dermique, mais aussi pulmonaire, que chez les souris sauvages. Il est à noter qu'une moindre fibrose hypodermique est également observée dans le modèle génétique de sclérodermie des souris $T s k{ }^{5}$ invalidées pour le gène $t / r 4$, suggérant des relations entre TLR-4 et voies de fibrose indépendantes de l'inflammation, le modèle Tskl étant dû à des mutations touchant la fibrilline qui active les voies du TGF $\beta[13,33]$.

Les tissus cutanés lésés sont infiltrés par différentes cellules immunitaires: des lymphocytes $\mathrm{T} \mathrm{CD}^{+}$, des macrophages, des lymphocytes B activés, des cellules dendritiques plasmacytoïdes et des mastocytes. Parmi les lymphocytes $C D 4^{+}$, les cellules de type Th2 (T helper 2), caractérisées par une prédominance de production d'IL-4 et d'IL-13, prédominent sur les cellules de type Thl (Figure 2). Le rôle éventuel des cellules Th17 reste à explorer, les résultats des travaux in vitro (effets pro-inflammatoire et antifibrosant sur les fibroblastes) et

${ }^{5}$ Le phénotype des souris hétérozygotes $T s k I^{1+}$ (qui développent spontanément une fibrose hypodermique, un emphysème pulmonaire, une autoimmunité mais sans atteinte vasculaire) est dû à une duplication partielle en phase du gène $F b n l$ codant la fibrilline-1, aboutissant à la synthèse d'une protéine avec un poids moléculaire augmenté. in vivo (actions antifibrosantes) étant contradictoires. Différentes approches suggèrent que la plasticité des macrophages puisse intervenir dans le développement des lésions. La polarisation M2 semble prédominer dans la SSc. Elle se caractérise par une sécrétion faible d'IL-12 et d'IL-23, et élevée d'IL-10, par l'expression de différents marqueurs de surface (CD163, scavenger receptor cysteine-rich type 1 protein $\mathrm{M130}$ ), et par une synthèse accrue de cytokines telles que I'IL-4, I'IL-10, I'IL-13 ou I'IL-6, de CTGF (connective tissue growth factor), mais aussi d'enzymes comme l'arginase-1 qui peut stimuler la synthèse de collagène [12].

\section{Cytokines et chimiokines}

Les cytokines Th2 jouent un rôle important dans la physiopathologie de la SSc ; leurs taux sont en effet augmentés dans la circulation et les tissus lésés au cours de la SSc. Elles activent la prolifération des fibroblastes et augmentent leur capacité de synthèse de composants de la matrice extracellulaire [14].

L'IL-6 a fait l'objet de travaux récents, stimulés notamment par la mise à disposition de biothérapies antiIL-6. Cette cytokine joue un rôle central dans la défense de l'hôte grâce à son action sur les systèmes immunitaire et hématopoiétique, en particulier lors de la phase aiguë de l'inflammation. Elle agit sur son récepteur (formé du complexe entre les chaînes IL-6R $\alpha$ et gp130) qui, via l'activation du système JAK (janus kinases)/ STAT (signal transducers and activators of transcription), induit la transcription de nombreux gènes contribuant aux réponses inflammatoire mais aussi immunitaire de type Th2, celle-ci étant dominante. Dans les modèles animaux, une invalidation du gène codant I'IL-6, ou l'inhibition de l'action de la cytokine, préviennent la fibrose induite (ou déjà établie) par la bléomycine. Elles réduisent également les fibroses cutanée et pulmonaire dans le modèle d'immunisation à la topo-isomérase ${ }^{6}$ [15]. En revanche, bloquer I'IL-6 est inefficace dans le modèle non inflammatoire des souris Tsk1. Ces résultats issus de modèles précliniques, ainsi que les données obtenues chez l'homme basées sur des mesures dans le sérum ou les tissus, suggèrent que le blocage de la voie de l'IL-6 devrait être appliqué aux stades précoces de la maladie, quand l'inflammation est active [16]. Un essai clinique de phase 2 évaluant le tocilizumab (un anticorps monoclonal dirigé contre la sous-unité IL-6R $\alpha$ du récepteur de l'IL-6) dans la sclérodermie a montré une tendance bénéfique sur le

${ }^{6}$ Une immunisation après injection de topo-isomérase de type I et d'adjuvant complet de Freund induit chez la souris une fibrose dermique et pulmonaire ainsi que la production d'autoanticorps spécifiques de la SSc. 
score cutané et également un signal positif pulmonaire. Une étude de phase 3 (NCT02453256) est en cours.

Les chimiokines ont un rôle important dans l'angiogenèse, la cicatrisation et la fibrose. Chez les patients atteints de SSc, les concentrations circulantes et tissulaires de CCL2 ( $C-C$ motif chemokine 2 ou MCP-1) sont augmentées de même que celles de CCL18, CCL3 et IL-8. Les données les plus convaincantes impliquent CCL2 dans la fibrose dermique, et CCL18 dans la fibrose pulmonaire [17]. Les cellules dendritiques plasmacytoïdes isolées des patients sécrètent des quantités élevées d'une chimiokine initialement décrite pour les plaquettes (PF4 [platelet factor 4] ou CXCL4 [C-X-C motif ligand 4]). Des taux élevés de cette chimiokine au niveau sanguin et dans la peau seraient prédictifs de la progression de la maladie vers des formes sévères intégrant une fibrose pulmonaire ou une hypertension pulmonaire [18].

\section{Autoanticorps}

La présence d'autoanticorps est depuis longtemps utilisée pour identifier et stratifier les malades atteints de SSc. Ce paramètre vient d'être intégré aux critères de classification indiqués plus haut. Ces autoanticorps, dirigés contre des autoantigènes ubiquitaires, nucléaires et nucléolaires (protéine centromérique B, topo-isomérase I, ARNpolymérase III, etc.), ségrégent avec certains sous-types cliniques. Toutefois leur rôle pathogène reste incertain même si certains travaux ont pu suggérer leur implication dans l'activation des fibroblastes, notamment pour les anti-topo-isomérase I [19]. D'autres autoanticorps, qui ne reconnaissent pas des autoantigènes ubiquitaires, ont été décrits plus récemment. Ils sont dirigés contre les MMPl ou MMP3 (métalloprotéases), les protéines de choc thermique (HSP47 [heat choc protein 47]) et la fibrilline-1.

Des autoanticorps, réagissant avec le récepteur du PDGF (platelet derived growth factor) exprimé sur les fibroblastes, et activant ces cellules, ont également été décrits chez des patients atteints de SSc mais aussi chez des patients présentant une GVHD ([graft versus host disease], réaction du greffon contre l'hôte). Le mécanisme impliquant ces anticorps est extrêmement séduisant pour expliquer la fibrose, mais les résultats montrant leur action possible n'ont pas pu être reproduits par des laboratoires indépendants [20]. Plus récemment, des autoanticorps antirécepteurs à l'endothéline et à l'anti-angiotensine 2 ont été mis en évidence chez les patients; ils pourraient être utiles comme biomarqueurs prédictifs de certaines complications vasculaires [21].

Les autoanticorps anti-ARN pol III sont assez rares. Ils sont observés dans une forme particulière de la maladie, forme cutanée diffuse avec risque élevé de complication rénale appelée «crise rénale ». Chez des patients atteints de SSc et d'un cancer, chez lesquels de tels autoanticorps avaient pu être détectés, des mutations somatiques du gène POLR3 (codant la polymérase) ont été décrites. Elles ne sont jamais présentes chez les patients n'ayant pas d'autoanticorps antiARN pol III. Lorsque ces mutations de POLR3 existent, des cellules $T$ qui reconnaissent le peptide correspondant à la protéine POLR3 mutée peuvent être détectées. Ces cellules T expriment une spécificité restreinte au patient, au peptide et au groupe HLA (CMH, complexe majeur d'histocompatibilité humain) [22]. La SSc pourrait ainsi être considérée, parfois, comme un syndrome paranéoplasique, déclenché par l'exposition à un néoantigène tumoral possédant une réactivité croisée avec des molécules endogènes.

\section{Mécanismes de formation et caractéristiques de la fibrose dans la SSc}

L'aboutissement de la séquence pathogénique de la SSc est la fibrose. Elle peut toucher différents organes mais débute habituellement au niveau du derme profond de la peau. Cette fibrose est définie par l'accumulation de matrice extracellulaire (MEC). Elle résulte d'une synthèse exagérée de composants de la MEC, mais des défauts de résorption et des modifications de sa composition et de son organisation contribuent à sa progression. Les dépôts de collagène, mais aussi d'acide hyaluronique, de glycosaminoglycanes ou de fibronectine, forment un tissu conjonctif épais et rigide qui détruit l'architecture d'origine et perturbe les fonctions du tissu. Le tissu fibreux est également caractérisé par l'apparition de myofibroblastes, des cellules positives pour l'alpha-actine du muscle lisse (alpha-SMA [smooth muscle actin]) et résistantes à l'apoptose. L'origine de ces myofibroblastes n'est pas clairement établie. Des fibroblastes résidents peuvent se différencier, de même que d'autres cellules résidentes comme les péricytes ou les cellules endothéliales. Des cellules progénitrices (fibrocytes) pourraient aussi être attirées et contribuer à l'accumulation des myofibroblastes. Une autre source de myofibroblastes a été évoquée avec l'implication possible de cellules progénitrices, normalement confinées dans la couche graisseuse adjacente au derme, qui pourraient se distribuer dans l'ensemble du derme lésionnel, perdre leurs marqueurs adipocytiques et exprimer des marqueurs de myofibroblastes [23].

\section{La voie du TGF $\beta$}

De nombreux médiateurs contribuent à l'activation fibroblastique observée dans la SSc. Le TGF $\beta$ est toutefois considéré comme le facteur régulateur central des processus de fibrose. L'activité du TGF $\beta$ implique trois sous-types de récepteurs et la voie Smad. Celle-ci est sous le contrôle de différents facteurs dont des inhibiteurs des $S$ mad $(S \operatorname{mad} 7$ ) et aussi le récepteur nucléaire NR4Al (nuclear receptor subfamily 4, group a, member 1) qui régule la dégradation de Smad7. Un travail a mis en lumière un rôle original du récepteur nucléaire NR4Al dans la SSc. Lors d'un processus physiologique comme la cicatrisation, NR4Al, induit par le TGF $\beta$, exerce un rétrocontrôle négatif prévenant les conséquences 
délétères d'une exposition trop longue au TGF $\beta$. En revanche, en cas de fibrose, l'activité persistante du TGF $\beta$ inhibe cette boucle de rétrocontrôle via des régulations transcriptionnelles et post-transcriptionnelles [24]. L'utilisation de petites molécules agonistes de NR4Al a permis de pallier le défaut d'activité du facteur et d'inhiber le développement de la fibrose, dans des modèles d'atteintes cutanées mais aussi pulmonaires, hépatiques et rénales. Ces résultats ouvrent des perspectives thérapeutiques innovantes pour ce groupe de maladies. La signalisation TGF $\beta$ peut également emprunter des voies non canoniques dont plusieurs ont été mises en évidence au cours de la SSc.

D'autres récepteurs nucléaires interviennent dans la fibrose. Les récepteurs $X$ hépatiques (pregnane $X$ receptor, PXR) modulent l'infiltrat inflammatoire et la synthèse de cytokines. D'autres, comme le récepteur de la vitamine $D$ ou les proliférateurs de peroxysomes (peroxisome proliferator-activated receptor, PPAR) peuvent directement agir sur le fibroblaste [25]. Certains médiateurs peuvent transduire le signal profibrosant du TGF $\beta$, tels que le facteur transcriptionnel EGRl (early growth response 1), la tyrosine kinase Abelson ( $A B L 1$ ou $c-A B L$ ), FAK (focal adhesion kinase), la répression de FLIl (friend leukaemia integration 1 ) et KLF (krüppel-like factor). En particulier, il existe une activation constitutive de FAK dans les fibroblastes issus de malades avec SSc : cette protéine étant à la croisée des voies d'activation du TGF $\beta$ et des voies de mécanotransduction induites par différentes intégrines, elle peut influencer la différenciation des fibroblastes en myofibroblastes et la production d'espèces réactives de l'oxygène (Figure 2).

\section{La voie du CTGF}

Le CTGF (connective tissue growth factor ou CCN2) est une protéine matricielle de la famille des CCN (connective tissue growth factor [CTGF], cystein rich protein [Cyr61], et nephroblastoma overexpressed gene). Elle est synthétisée par les cellules endothéliales et est impliquée dans de multiples fonctions cellulaires ainsi que dans différents cancers. Induit par le TGF $\beta$, l'endothéline 1 et l'angiotensine II, le CTGF promeut, en partenariat avec le TGF $\beta$, le processus de fibrose. II agit avec le PDGF (platelet-derived growth factor) qui est un puissant facteur mitogène des cellules mésenchymateuses. Le PDGF est produit par les cellules endothéliales, les plaquettes, les macrophages et les fibroblastes. Ses récepteurs sont fortement exprimés dans la peau et les poumons des malades atteints de SSc.

D'autres acteurs, que nous ne ferons que citer, ont été mis en évidence dans la SSc : les espèces réactives de l'oxygène (ROS), l'infiltrat inflammatoire mais aussi les phénomènes d'ischémie/reperfusion et d'hypoxie chronique qui pourraient contribuer au stress oxydant observé dans la SSc. La voie Wnt, lorsque son activation est dérégulée, peut aussi induire la différenciation des fibroblastes en myofibroblastes et ainsi contribuer à la fibrose. Toutes ces voies pourraient être des cibles pour des molécules inhibitrices, potentiellement intéressantes, utilisées dans des approches précliniques [26].

II ne faut pas oublier dans ce tableau les facteurs mécaniques qui interviennent dans l'activation et la prolifération fibroblastique. Ils mettent en jeu des intégrines de surface et également des tensions dans le cytosquelette qui activent différentes kinases. Dans les modèles murins, des mutants de la fibrilline (le principal composant des microfibrilles de la matrice extracellulaire du derme), ou d'intégrines liant la fibrilline, développent une fibrose dermique proche de celle décrite dans la SSc. Ils présentent une inflammation cutanée et un profil d'autoanticorps très spécifique de la SSc. Le phénotype de ces souris peut être modifié par l'administration d'anticorps neutralisant le TGF $\beta$ ou, à l'inverse, par des anticorps activant les intégrines alpha-1 [27].

\section{Facteurs génétiques associés à la SSc}

Des données convergentes ont montré une susceptibilité génétique dans la SSc. II existe ainsi un sur-risque familial : risque de récurrence proche de 13 pour les apparentés de premier degré et de 15 pour les frères et sœurs ${ }^{7}$. Cependant, une étude réalisée sur des jumeaux (42 paires dont 24 monozygotes) a montré une faible concordance pour l'expression clinique de la maladie $(4,7 \%)$. Il existe cependant une forte corrélation entre la présence d'anticorps antinucléaires et le profil d'expression génique de fibroblastes dermiques en culture [28].

\section{Polymorphismes nucléotidiques simples}

Des études d'association gène-candidat, puis de génome entier (genome-wide association study, GWAS) et par immuno-chip (puces à ADN) ont mis en évidence un certain nombre de gènes de susceptibilité associés à la maladie. Différents polymorphismes nucléotidiques simples (SNP, single nucleotidic polymorphisms) dans le complexe majeur d'histocompatibilité (CMH) ont en effet été identifiés, avec des associations surtout liées aux différents autoanticorps exprimés. Hormis celles définies pour le $\mathrm{CMH}$, les associations les plus robustes portent sur des gènes impliqués dans les régulations de l'immunité et les plus solidement identifiés concernent IRF5 (interferon regulatory factor 5) et STAT4 (signal transducer and activator of transcription 4) [29, 30]. Toutefois, les variants sont la plupart du temps situés dans des régions non codantes des gènes et sans prédiction de fonction régulatrice. Ainsi, aucun variant causal n'est identifié et les conséquences biologiques des variants restent incertaines. De plus, la plupart des gènes mis en évidence sont également associés à d'autres maladies autoimmunes plus ou moins proches de la SSc, définissant ce que l'on appelle « l'auto-

\footnotetext{
${ }^{7}$ Soit un sur-risque de développer la maladie multiplié par 15 chez les frères et
} sœurs d'un cas et multiplié par 13 pour les apparentés de $1^{\text {re }}$ génération. 
immunité partagée ». Une des questions clés qui demeure sans réponse est de comprendre la raison pour laquelle des maladies telles que la SSc, le lupus systémique ou les thyroïdites, bien qu'ayant des bases génétiques proches, s'expriment par des phénotypes si différents. De nouvelles études seront donc nécessaires pour mieux identifier les facteurs génétiques en cause, leur architecture exacte (épistasie), leur rôle et leurs interactions avec les facteurs d'environnement.

\section{Épigénétique}

La contribution modeste des facteurs génétiques identifiés à ce jour a conduit à explorer le rôle des modifications épigénétiques qui influencent l'expression des gènes sans modifier le code génétique $(\rightarrow)$. Une approche de séquençage à large échelle de la méthylation de l'ADN sur l'ensemble du génome, réalisée à partir de la culture de fibroblastes, a révélé de grandes différences de méthylation

\section{$(\rightarrow)$ Voir le numéro} thématique DOHaD, $m / s n^{\circ} 1$, janvier 2016 des îlots $\mathrm{CpG}^{8}$, entre des patients présentant une SSc et des sujets contrôles. Par ailleurs, deux facteurs transcriptionnels participant aux régulations épigénétiques (KLF5 [kruppel-like factor 5] et FLI] [friend leukemia integration 1]) ont été identifiés chez les patients. Ils sont impliqués dans la SSc en modulant l'expression de gènes de fibrose, ce qui est conforté par des études réalisées dans des modèles de souris transgéniques dans lesquels l'inactivation de ces deux gènes se traduisait par le phénotype particulier de la SSc [31]. Des excès de méthylation ont également été observés au niveau de différents gènes régulant les fonctions des fibroblastes dont plusieurs acteurs de la voie Wnt : DKK (dickkopf WNT signalling pathway inhibitor 1), WIFl (WNT inhibitory factor 1), SFRPl (secreted frizzled-related protein 1). Des modifications de méthylation de I'ADN de certains gènes exprimés par les lymphocytes T CD4 ${ }^{+}$ont aussi été mis en évidence : DNMT1 (DNA cytosine-5-methyl-transferase 1) et FOXP3 (forkhead box P3). De même, des modifications post-traductionnelles des histones ont été suggérées, impliquant les HDAC (histone deacetylases) dans la réponse profibrosante dans plusieurs modèles.

Les micro-ARN (miRNA) sont des ARN non codants constitués de 18 à 23 nucléotides qui interviennent dans la régulation de l'expression des gènes. Dans la SSc, un rôle possible des miR-21 (micro-ARN 21) et miR-29 a été décrit. Plus récemment, l'activité antifibrosante de miR-193b, par une action notamment sur le remodelage vasculaire et impliquant uPA (urokinase-type plasminogen activator) et le TGF $\beta$, dans sa régulation, a été mise en évidence in vitro et in vivo, dans différents modèles animaux [32].

\section{Conclusion}

Les nombreux progrès réalisés dans la compréhension des mécanismes cellulaires et moléculaires de la séquence pathogénique de la SSc laissent espérer le développement de futures interventions thérapeutiques permettant réellement de prévenir ou de réduire les lésions fibrosantes qui caractérisent cette maladie. $\diamond$

\footnotetext{
${ }^{8}$ Zones riches en dinucléotide CG, cibles de la méthylation.
}

\section{SUMMARY}

Pathophysiology of systemic sclerosis

Systemic sclerosis (SSc) is an orphan disease affecting the connective tissue. The cause of SSc remains unknown but is likely to involve environmental factors in a genetically primed individual with SSc belonging to the multigenic disorders. Pathogenesis is dominated by early microvascular changes targeting endothelial cells and with the release of several mediators promoting an inflammatory response and vascular remodelling. Several lines of evidence position autoimmunity as a key perpetuating event with activation of both innate and adaptive immunity and with the production of distinct autoantibodies. The cascade ultimates with the fibrosis defined by accumulation of extra-cellular matrix through an imbalance between synthesis and degradation of several components and mesenchymal cell activation and differentiation controlled by a large number of autocrine and paracrine factors. $\diamond$

\section{LIENS D'INTÉRÊT}

L'auteur déclare participer à des interventions ponctuelles pour les entreprises Roche, Bayer, Inventiva, Medac, Boehringer, Actelion et Sanofi.

\section{REMERCIEMENTS}

Inserm, programme ATIP/AVENIR, Association des sclérodermies de France.

\section{RÉFÉRENCES}

1. Van den Hoogen F, Khanna D, Fransen J, et al. 2013 classification criteria for systemic sclerosis: an American college of rheumatology/European league against rheumatism collaborative initiative. Ann Rheum Dis 2013 ; 72 : 1747-55.

2. Elhai M, Meune C, Avouac J, et al. Trends in mortality in patients with systemic sclerosis over 40 years: a systematic review and meta-analysis of cohort studies. Rheumatology (Oxford) 2012 ; 51 : 1017-26.

3. Elhai M, Avouac J, Walker UA, et al. A gender gap in primary and secondary heart dysfunctions in systemic sclerosis: a EUSTAR prospective study. Ann Rheum Dis 2014 ; doi: 10.1136/annrheumdis-2014-206386.

4. Matucci-Cerinic M, Kahaleh B, Wigley FM. Review: evidence that systemic sclerosis is a vascular disease. Arthritis Rheum 2013 ; 65 : 1953-62.

5. Rabquer BJ, Hou Y, Del Galdo F, et al. The proadhesive phenotype of systemic sclerosis skin promotes myeloid cell adhesion via ICAM-1 and VCAM-1. Rheumatology (Oxford) $2009 ; 48: 734-40$.

6. Liu S, Parapuram SK, Leask A. Fibrosis caused by loss of PTEN expression in mouse fibroblasts is crucially dependent on CCN2. Arthritis Rheum 2013 ; $65: 2940-4$.

7. Perros F, Humbert M, Cohen-Kaminsky S. Hypertension artérielle pulmonaire. Med Sci (Paris) $2013 ; 29: 607-16$.

8. Ghatnekar A, Chrobak I, Reese C, et al. Endothelial GATA-6 deficiency promotes pulmonary arterial hypertension. Am J Pathol 2013 ; 182 : 2391-406.

9. Derrett-Smith EC, Dooley A, Gilbane AJ, et al. Endothelial injury in a transforming growth factor $\beta$-dependent mouse model of scleroderma induces pulmonary arterial hypertension. Arthritis Rheum 2013 ; 65 : 2928-39.

10. Maurer B, Distler A, Suliman YA, et al. Vascular endothelial growth factor aggravates fibrosis and vasculopathy in experimental models of systemic sclerosis. Ann Rheum Dis 2014 ; 73 : 1880-7.

11. Kuwana M, Okazaki Y. Brief report: impaired in vivo neovascularization capacity of endothelial progenitor cells in patients with systemic sclerosis. Arthritis Rheum 2014 ; 66 : 1300-5. 


\section{RÉFÉRENCES}

12. Christmann RB, Sampaio-Barros P, Stifano G, et al. Association of interferon- and transforming growth factor $\beta$-regulated genes and macrophage activation with systemic sclerosis-related progressive lung fibrosis. Arthritis Rheum 2014 ; 66 : 714-25.

13. Takahashi T, Asano Y, Ichimura $Y$, et al. Amelioration of tissue fibrosis by toll-like receptor 4 knockout in murine models of systemic sclerosis. Arthritis Rheum $2015 ; 67: 254-65$.

14. Fuschiotti P, Larregina AT, Ho J, et al. Interleukin-13-producing CD8 ${ }^{+} T$ cells mediate dermal fibrosis in patients with systemic sclerosis. Arthritis Rheum $2013 ; 65$ : 236-46.

15. Yoshizaki A, Yanaba K, Ogawa A, et al. Immunization with DNA topoisomerase I and Freund's complete adjuvant induces skin and lung fibrosis and autoimmunity via interleukin- 6 signaling. Arthritis Rheum $2011 ; 63: 3575-85$.

16. Desallais L, Avouac J, Fréchet M, et al. Targeting IL-6 by both passive or active immunization strategies prevents bleomycin-induced skin fibrosis. Arthritis Res Ther $2014 ; 16$ : R157.

17. Assassi S, Wu M, Tan FK, et al. Skin gene expression correlates of severity of interstitial lung disease in systemic sclerosis. Arthritis Rheum 2013 ; 65 : 2917-27.

18. Van Bon L, Affandi AJ, Broen J, et al. Proteome-wide analysis and CXCL4 as a biomarker in systemic sclerosis. N Engl J Med 2014 ; $370: 433-43$.

19. Arcand J, Robitaille G, Koenig M, et al. Heparin inhibits the interaction of DNA topoisomerase I/ anti-topoisomerase I immune complexes with heparan sulfate on dermal fibroblasts. Arthritis Rheum $2012 ; 64: 1632-41$.

20. Moroncini G, Grieco A, Nacci G, et al. Epitope specificity determines pathogenicity and detectability of anti-PDGFR $\alpha$ autoantibodies in systemic sclerosis. Arthritis Rheum $2015 ; 67$ : 1891-903.

21. Günther J, Kill A, Becker MO, et al. Angiotensin receptor type 1 and endothelin receptor type $A$ on immune cells mediate migration and the expression of IL-8 and CCL18 when stimulated by autoantibodies from systemic sclerosis patients. Arthritis Res Ther $2014 ; 16:$ R65.

22. Joseph CG, Darrah $\varepsilon$, Shah AA, et al. Association of the autoimmune disease scleroderma with an immunologic response to cancer. Science $2014 ; 343: 152-7$.

23. Marangoni RG, Korman BD, Wei J, et al. Myofibroblasts in murine cutaneous fibrosis originate from adiponectin-positive intradermal progenitors. Arthritis Rheum 2015; $67: 1062-73$.
24. Palumbo-Zerr K, Zerr P, Distler A, et al. Orphan nuclear receptor NR4Al regulates transforming growth factor $-\beta$ signaling and fibrosis. Nat Med $2015 ; 21: 150-8$.

25. Wei J, Zhu $H$, Komura K, et al. A synthetic PPAR- $\gamma$ agonist triterpenoid ameliorates experimental fibrosis: PPAR- $\gamma$-independent suppression of fibrotic responses. Ann Rheum Dis 2014 ; 73 : 446-54

26. Distler A, Ziemer C, Beyer C, et al. Inactivation of evenness interrupted (EVI) reduces experimental fibrosis by combined inhibition of canonical and noncanonical Wnt signalling. Ann Rheum Dis 2014 ; 73 : 624-7.

27. Gerber $\varepsilon \varepsilon$, Gallo $\varepsilon M$, Fontana SC, et al. Integrin-modulating therapy prevents fibrosis and autoimmunity in mouse models of scleroderma. Nature $2013 ; 503: 126-30$.

28. Dieudé P, Boileau C, Allanore Y. Immunogenetics of systemic sclerosis. Autoimmun Rev $201 ; 10: 282-90$.

29. Radstake TR, Gorlova 0, Rueda B, et al. Genome-wide association study of systemic sclerosis identifies CD247 as a new susceptibility locus. Nat Genet $2010 ; 42$ : 426-9.

30. Allanore $Y$, Saad M, Dieudé $P$, et al. Genome-wide scan identifies TNIP1, PSORSICl, and RHOB as novel risk loci for systemic sclerosis. PLoS Genet $2011 ; 7$ : el002091

31. Noda S, Asano Y, Nishimura S, et al. Simultaneous downregulation of KLF5 and Fli1 is a key feature underlying systemic sclerosis. Nat Commun $2014 ; 5: 5797$.

32. Iwamoto N, Vettori S, Maurer B, et al. Downregulation of miR-193b in systemic sclerosis regulates the proliferative vasculopathy by urokinasetype plasminogen activator expression. Ann Rheum Dis $2016 ; 75: 303-10$.

33. Wipff J, Allanore $Y$, Boileau C. Interactions entre la fibrilline- 1 et le TGF- $\beta$. Med Sci (Paris) $2009 ; 25: 161-7$

\section{TIRÉS À PART}

Y. Allanore

D ossédées du malin au Moyen-Âge, les sorcières hystériques sont vouées au bûcher. Enfermées au xvII siècle, maltraitées, elles rejoignent la Cour des Miracles de l'Hospice de la Vieillesse-Femmes à la Salpêtrière... Jusqu'à ce que le Dr JeanMartin Charcot (1825-1893) mène le combat qui transforme l'ancien hospice en hôpital : l'École de la Salpêtrière de

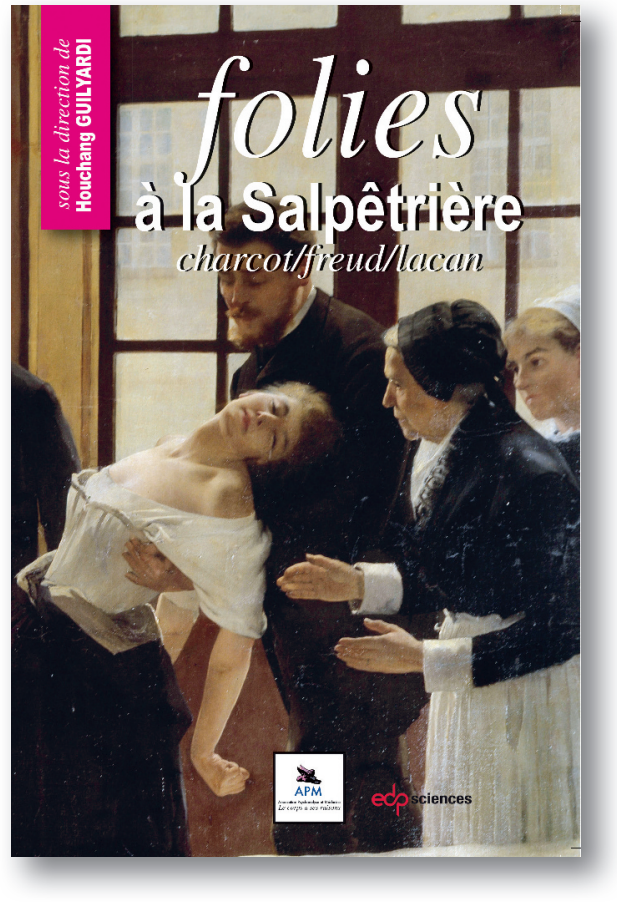

ISBN : 978-2-7598-1268-4

240 pages

$20 €$ Paris est née, qui devient lieu de recherche, d'enseignement et de soins, de renommée internationale.

Jean Martin Charcot n'a pas bonne presse, et pourtant... Hystérie et folie traversent les siècles, prenant les formes de «l'air du temps ».

De l'utérus migrateur d'Hippocrate aux recherches neurologiques de Charcot. Du désir inconscient avec Freud à la jouissance du parlêtre chez Lacan... C'est à cette traversée historique et conceptuelle que nous convie cet ouvrage.

À retourner à EDP Sciences, 109, avenue Aristide Briand, 92541 Montrouge Cedex, France Tél. : 0141177405 - Fax : 0149850345 - $\varepsilon$-mail : edk@edk.fr

NOM:

Adresse :

Code postal :

Pays :

Fonction :

Je souhaite recevoir

Folies à la Salpêtrière : $20 €+3 €$ de port $=23 € \pi T$

en .... exemplaire, soit un total de ....

$\square$ Par chèque, à l'ordre de EDP Sciences

$\square$ Par carte bancaire : $\quad \square$ Visa $\square$ Eurocard/Mastercard

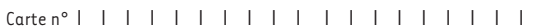

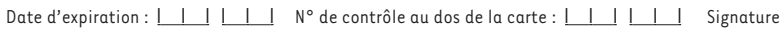

